\title{
Anti-HER2 Antibody-drug Conjugate MEDI4276
}

National Cancer Institute

\section{Source}

National Cancer Institute. Anti-HER2 Antibody-drug Conjugate MEDI4276. NCI

Thesaurus. Code C126355.

An antibody-drug conjug ate (ADC) composed of a bispecific antibody against the extracellular domain of human epidermal growth factor receptor 2 (HER2; ERBB2) comprised of the single-chain variable fragment (scFv) of the anti- HER2 monoclonal antibody trastuzumab, which binds to domain IV of HER2, fused to the heavy chains of the anti-HER2 monoclonal antibody 39S, which binds to domain II of HER2, and conjug ated, via a cleavable linker, to the cytotoxic anti-microtubule agent tubulysin, with potential antineoplastic activity. Upon administration of ADC MEDI4276, the anti-HER2 bispecific antibody specifically targets and binds to HER2 on the surface of certain cancer cells. Upon binding, crosslinking and internalization of antibody-HER2 complexes occurs and MEDI4276 is transported to the lysosome where the linker is cleaved, thereby delivering tubulysin inside HER2-expressing cancer cells. Tubulysin binds to tubulin and inhibits microtubule polymerization, which blocks cell division. This results in G2/M phase arrest, tumor cell apoptosis, and decreased proliferation of HER2-expressing tumor cells. HER2, a tyrosine kinase receptor, is overexpressed by many cancer cell types. 